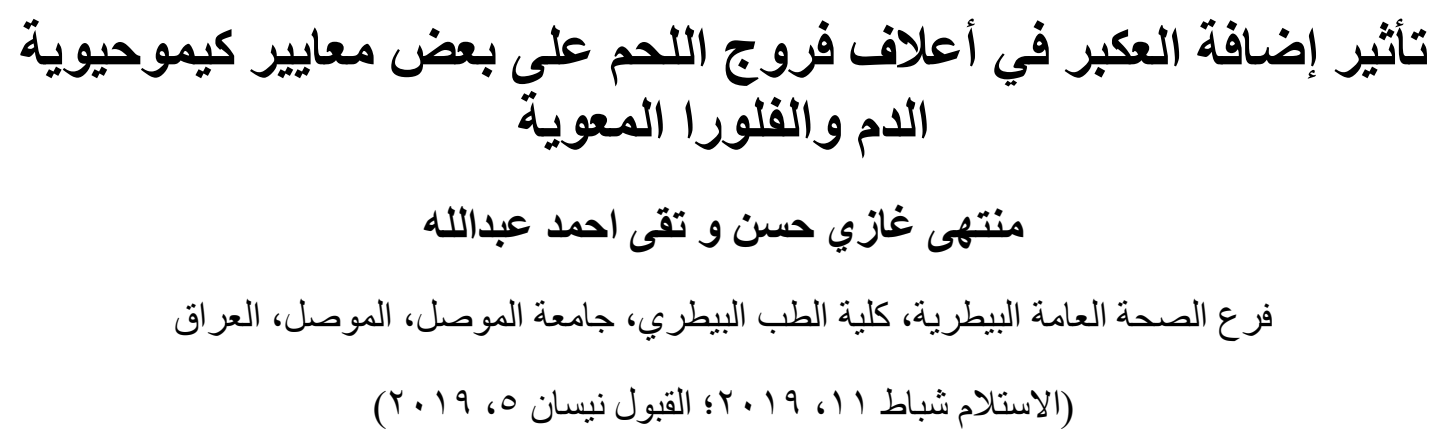

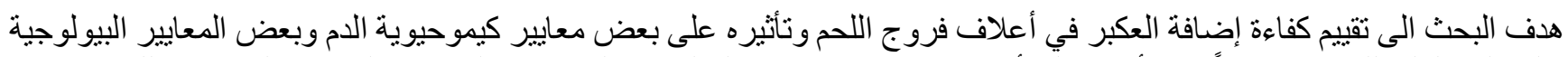

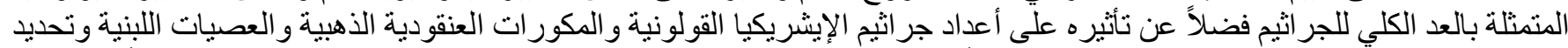

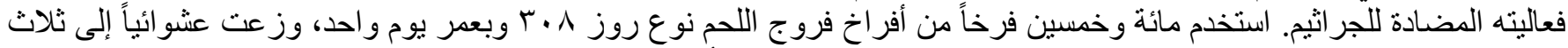

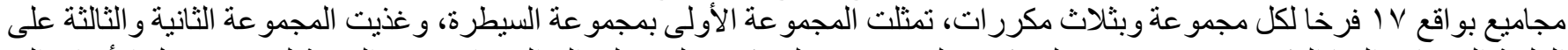

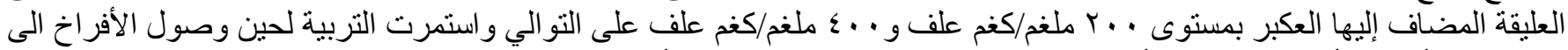

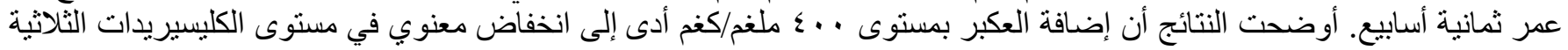

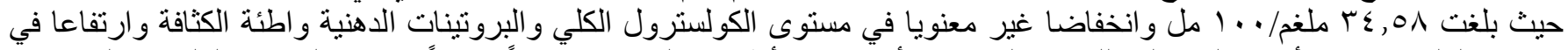

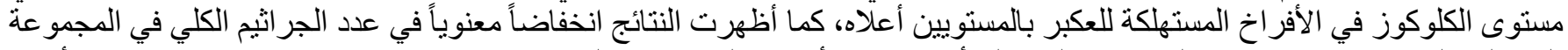

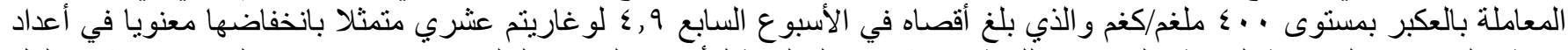

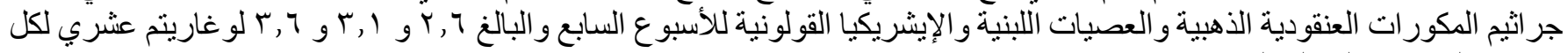
من هذه الجر اثيم على التو الي.

\title{
The effect of Propolis addition to broiler feeds on some blood biochemical parameters and intestinal flora
}

\author{
M.G. Hassan* and T.A. Abdullah \\ Department of Veterinary public health, College of Veterinary Medicine, University of Mosul, Mosul, Iraq \\ "Email: montaha_hassan99@yahoo.com
}

\begin{abstract}
The present study was conducted to evaluate the efficiency of adding Propolis to broiler feeds and the effect on some biochemical and biological parameters represented by its effect on the total bacterial count of intestinal flora as well as the antimicrobial effect on Escherichia coli, staphylococcus aureus and lactobacillus. We used 150 broiler chicks type Ross at the first day of age divided randomly into three groups each of 17 chicks with three replications, the first one is control group and the second and third group fed on feeds containing two levels of propolis $200 \mathrm{mg} / \mathrm{kg}$ and $400 \mathrm{mg} / \mathrm{kg} \mathrm{respectively.} \mathrm{Results} \mathrm{showed}$ that using propolis at $400 \mathrm{mg} / \mathrm{kg}$ in the diet lead to a significant decrease in level of triglycerides which was $34.58 \mathrm{mg} / 100 \mathrm{ml}$ accompanied by a mathematical reduction in total cholesterol and LDL levels, while there was an increase in glucose level. The highest propolis levels were effective in significant reduction in total bacterial count of intestinal flora, especially in the seventh week $4.9 \log _{10}$, represented by a significant reduction in counts of staphylococcus aureus, lactobacillus and Escherichia coli 2.6, 3.6, $3.1 \log _{10}$ respectively.
\end{abstract}

Keywords: Propolis, Antimicrobial effect, Broiler feed Available online at http://www.vetmedmosul.com, (C) 2020, College of Veterinary Medicine, University of Mosul. This is an open access article under the CC BY 4.0 license (http://creativecommons.org/licenses/by/4.0/). 
المواد الكورستين quercetin وحامض الكافائين caffeic acid وحامض الفريولك ferulic acid كما يقلل العكبر من تركيز

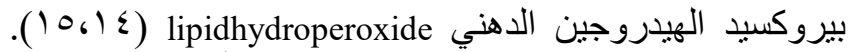

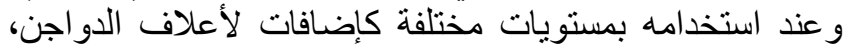

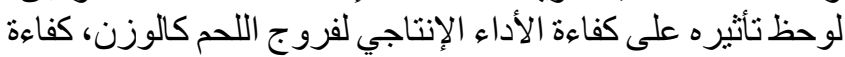

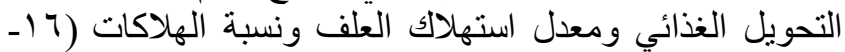

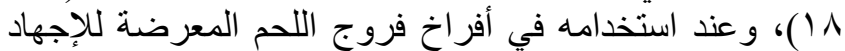

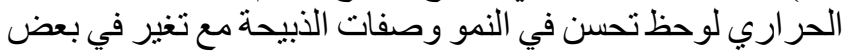

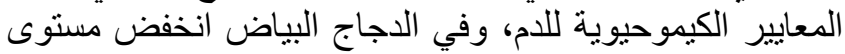

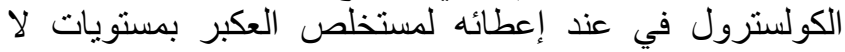

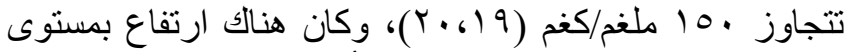

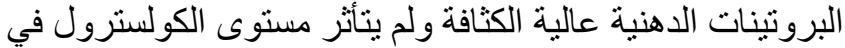
الدم وكذللك مستوى الكلسيريدات الثثلاثية و البروتين الكلي التئي (Y) (Y).

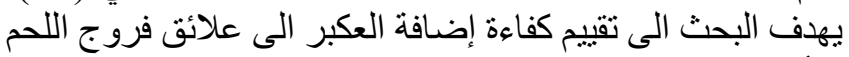

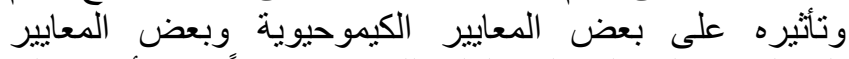
البايولوجية المتمثلة بالعد الكلي للجراثير فضلاً عن تأثيره على لئى

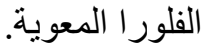

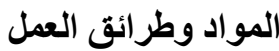

أجري البحث في الحقل الحيواني التابع لكلية الطب البيطري

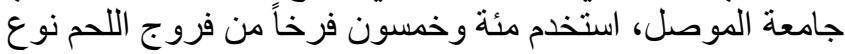

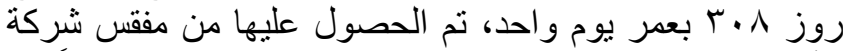

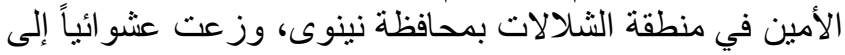

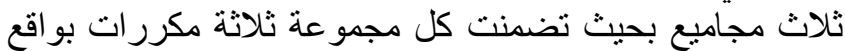

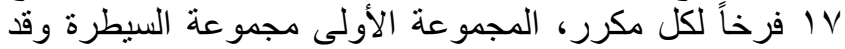

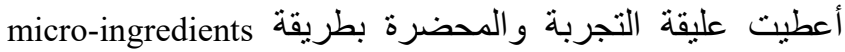
(الجدول ( ) من دون إضافة العكبر، المجموعة المبرة الثانية أعطيت

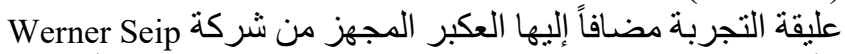

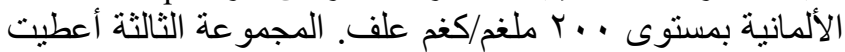

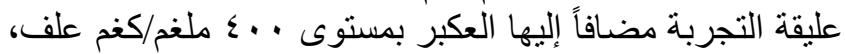

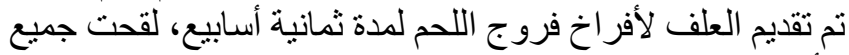

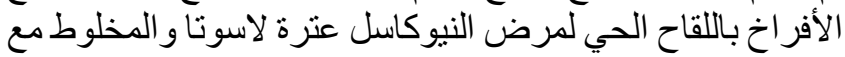

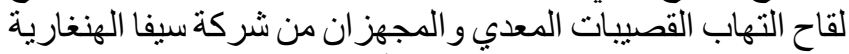

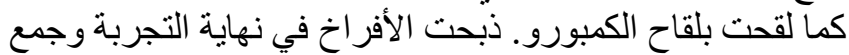

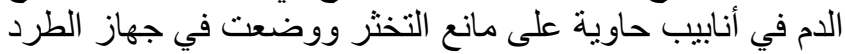

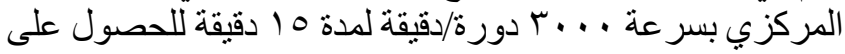

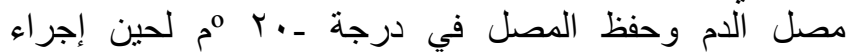
الفحوصات الكيموحيوية.

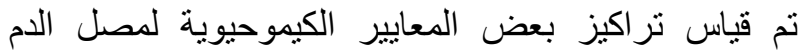

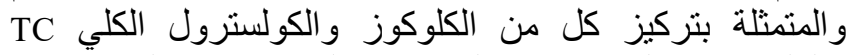

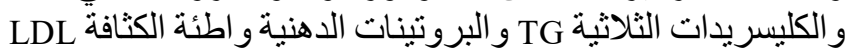

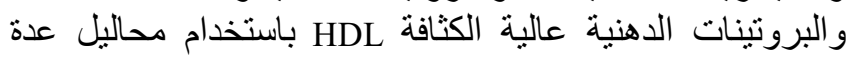

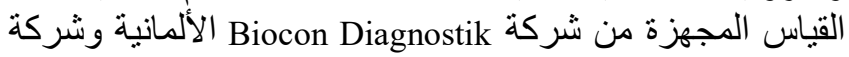
Biolabo

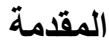

العكبر مادة اكتشفها الإغريق وأطلقوا عليها البروبولس تبعاً

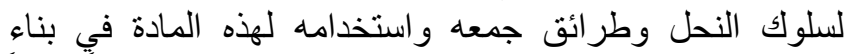

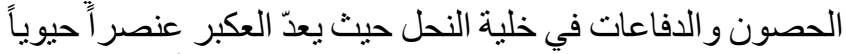

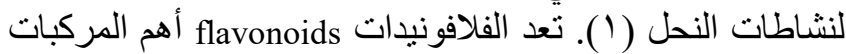
الرئيسة للعكبر وهي مركبات معقدة ذات أو اصر تصدات تهاهية قوية

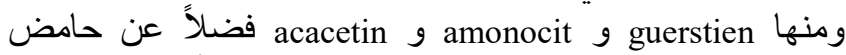

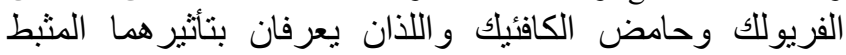

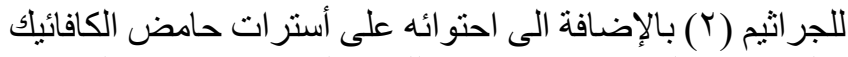

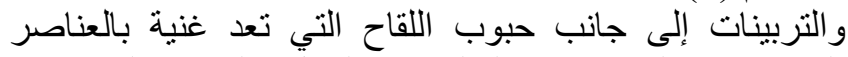

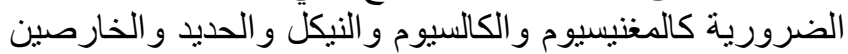

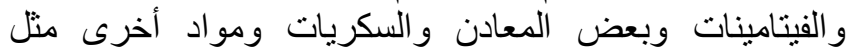

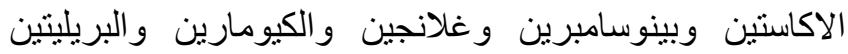

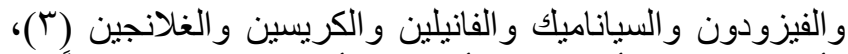

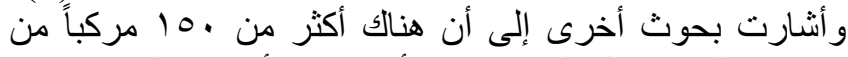

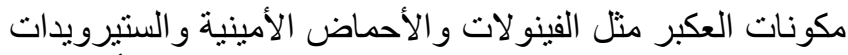

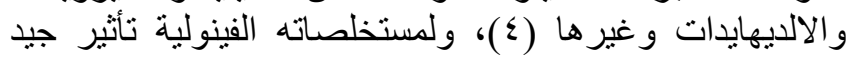

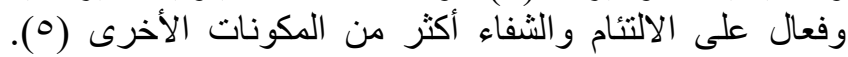

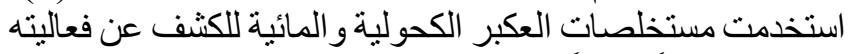

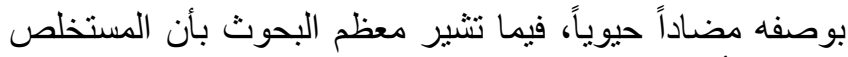

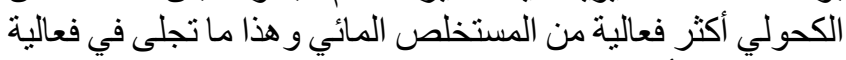

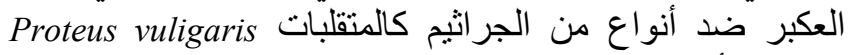
وبفعالية أقل ضد السالمونيلا S. pallorum و S. gallinarum و الإيشريكيا القولونية (7). تكون المكور ات العية العنقودية الذهبية أكثر

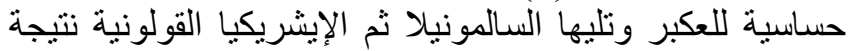

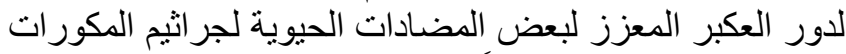

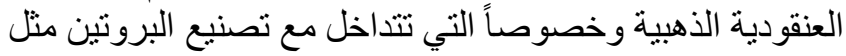
الكلورومفينيكول و الجنتامايسين و والفانكو مايسين و والتنر التهايكلين

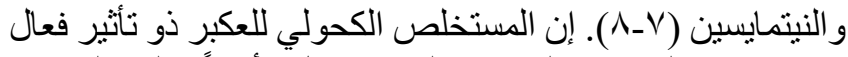

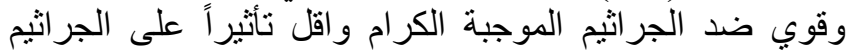

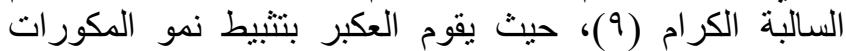

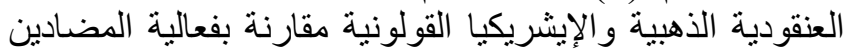

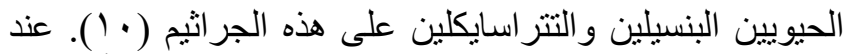

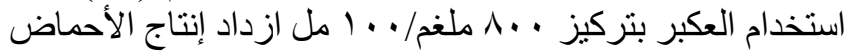

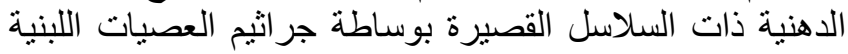
Lactobcillus acidophilus

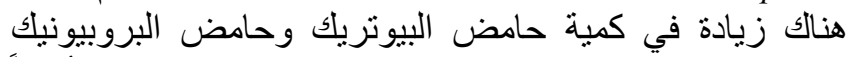

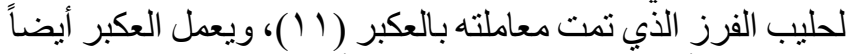

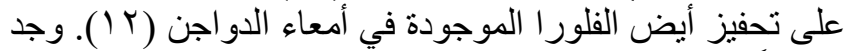

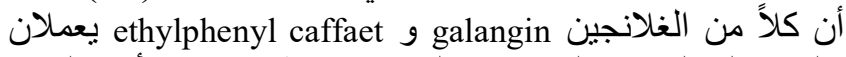

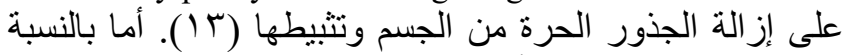

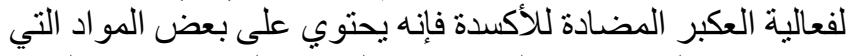

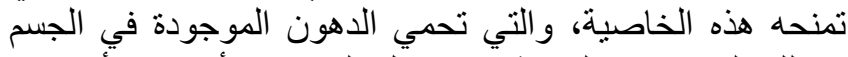
وكذلك البروتينات الدهنية في مصل الدام من الأكسدة، وأهم هذه اله 


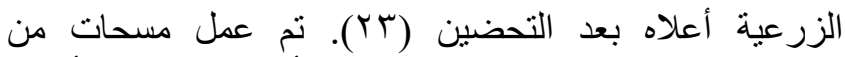
المستعمر ات النامية على كل من وسط الأيوسين مثيلين الأزرق

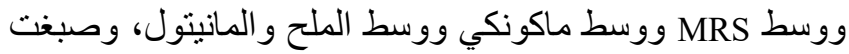

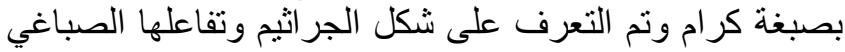

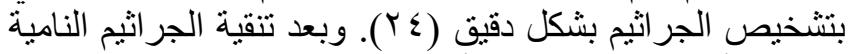

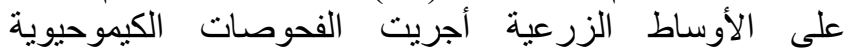

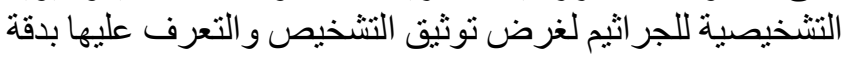

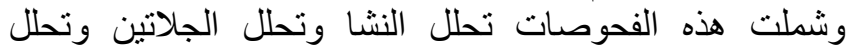

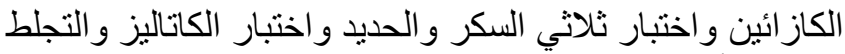

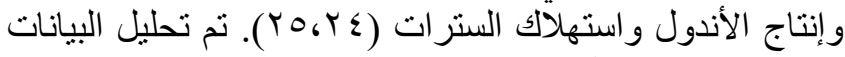

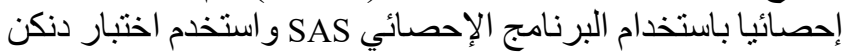

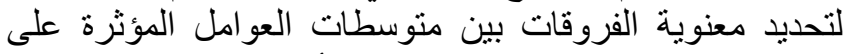

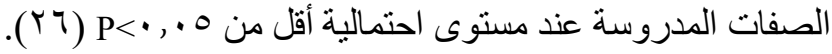

\section{النتائجج}

يوضح الجدول r نأثير العكبر على بعض المعايير الكيموحيوية

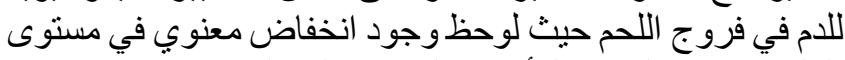

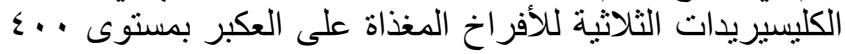

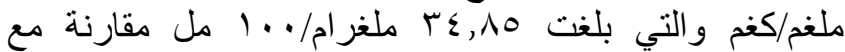

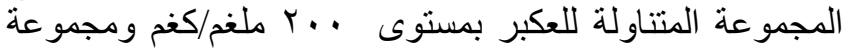

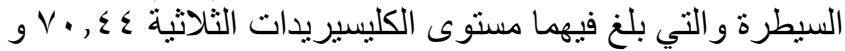

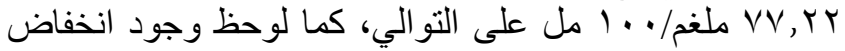

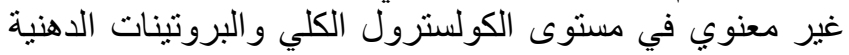

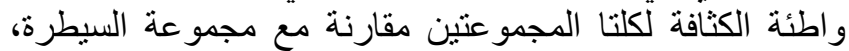

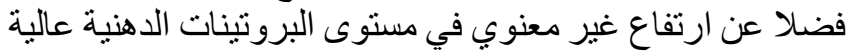

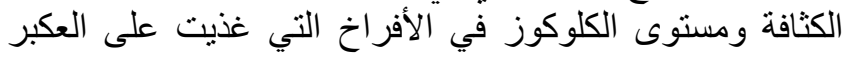

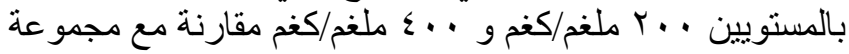

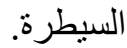

و بالا عتماد على الطريقة اللونية وباستخدام جهاز للمطياف الضوئي

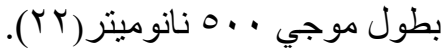

الجدول (: مكونات العليقة التي غُذيت عليها الأفر اخ خلال مدة

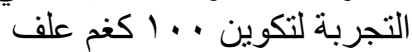

\begin{tabular}{|c|c|c|}
\hline العليقة الناهية & العليقة البادئة & المكونات \\
\hline . 7 كغم & 70 كغم & الحنطة المحلية \\
\hline . 1 كغم & - & شعير \\
\hline Y T Y T & rq,0 كغم & كسبة فول الصويا \\
\hline 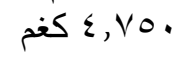 & r Sغم & زيت \\
\hline . Vo. 1, كغم & 1,V0. & فوسفات \\
\hline r, • كغم & r , • كغم & ملح الطعام \\
\hline ا كغم & ا كغم & فيتامينات ومعادن \\
\hline 1 , • كغم & $\cdot, 1$ & ميثيونين \\
\hline ا , • كغم &., .0 & أنزيمات \\
\hline$\%$ \% & $\%$ Yo & البروتين \\
\hline 99 19 וr كيلو & 101. & \\
\hline كالوري/كغم & كالوري/كغ & الطافه النمنيليه \\
\hline
\end{tabular}

كما جمعت نماذج من البراز بأخذ غرام واحد من البراز

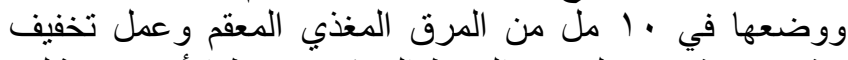
عشري ونشرت على في الوسط المغذي ووسط الأيوسين مثيلين المرين الأزرق Eosin Methylene Blue Agar ووسط فيط الماكونكي MRS agar MacConkey's Agar Mannitol Salt Agar ساعة وتم حساب عدد مستعمر ات الجر اثيم النامية على الأوساط لماط

$$
\text { الجدول ب: تأثير إضافة العكبر على بعض المعايير الكيموحيوية للام في فروج اللحم }
$$

\begin{tabular}{|c|c|c|c|c|c|}
\hline \multicolumn{5}{|c|}{ المعايير الكيموحيوية } & \multirow[b]{2}{*}{ المجاميع } \\
\hline ملغم/ديسليتز & 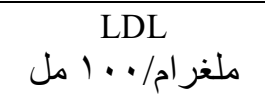 & 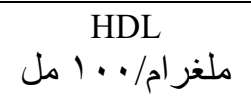 & 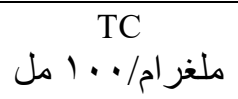 & 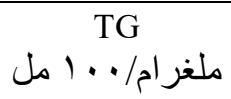 & \\
\hline a $r,, \varepsilon_{ \pm} r \wedge r, r$ & a $17, l_{ \pm} \mid \wedge \varepsilon, \wedge \varepsilon$ & $\mathrm{a} 9, l_{ \pm} \wedge \wedge, r$ & $\begin{array}{c}\mid V, \tau_{ \pm} r \tau T, r \varepsilon \\
a\end{array}$ & $\mathrm{a} \wedge, \tau_{ \pm} V \vee, Y r$ & السيطرة \\
\hline a $r_{0, r_{ \pm} r_{9}, r_{\tau}}$ & a $\left|V, o_{ \pm}\right| r r, r$ & $\left.\mathrm{a} \vee, q_{ \pm}\right) \cdot 1, \varepsilon$. & a $1 \leqslant, \tau_{ \pm} r r q, \tau$ & a $T, \Sigma \pm V \cdot, \Sigma \varepsilon$ & العكبر · . ب ملغم/كغم \\
\hline$a \leqslant 1, Y_{ \pm} \leqslant 0 \leqslant$ & a $\mid r r, q \cdot \pm r o, 1$ & a $\| \wedge, r \cdot \pm r, \tau$ & $r \cdot, \gamma_{ \pm} r r v, q \Lambda$ & $b r, V_{ \pm} r \varepsilon, O \wedge$ & ع ملغم/كغم \\
\hline
\end{tabular}

الحروف الصغيرة عموديا تمثل الاختلافات المعنوية عند مستوى احتمالية ه . , • P>.

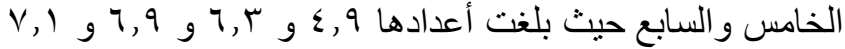

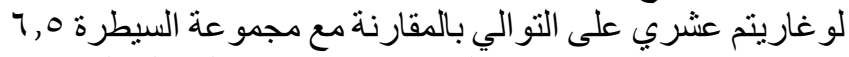

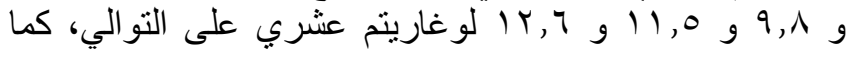

أوضحت النتائج المبينة في الجدول ب وجود انخفاض معنوي

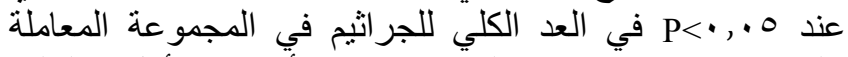

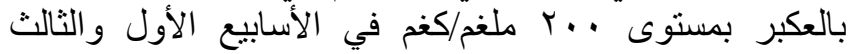


المعاملة بالعكبر بمستوى . . م ملغم/كغم وأفراخ مجموعة

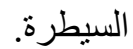

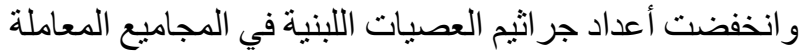

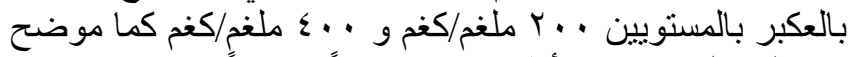

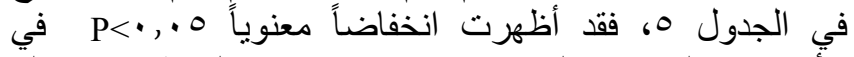

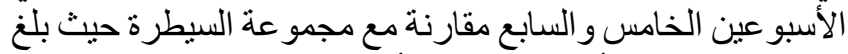

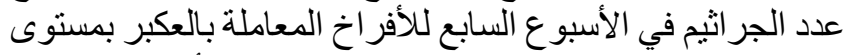

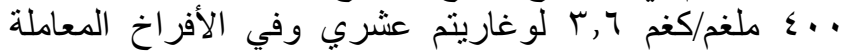

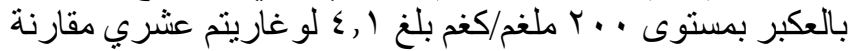

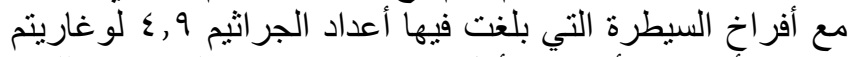

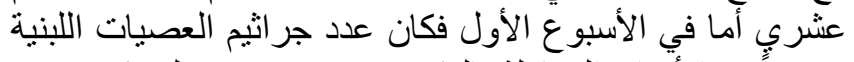

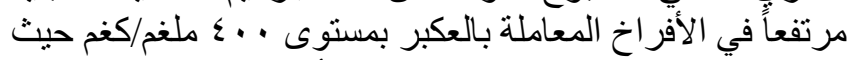

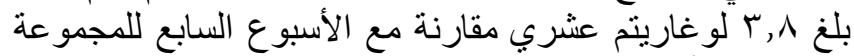

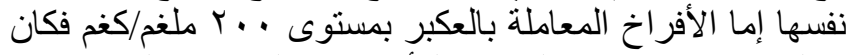

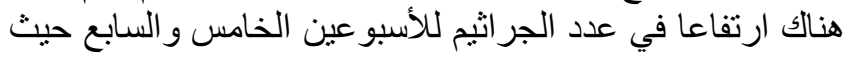

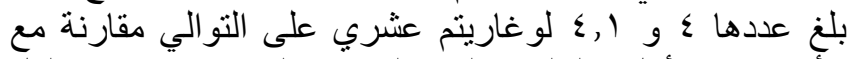

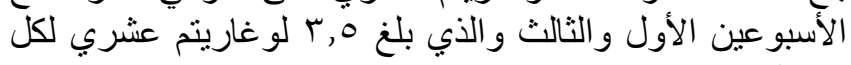

منهما.

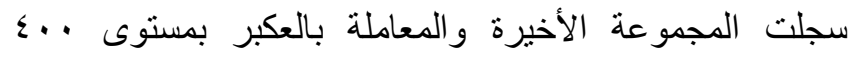

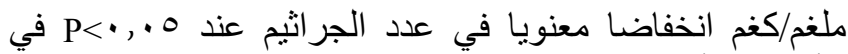

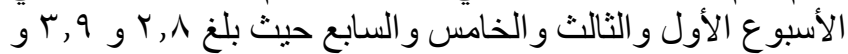

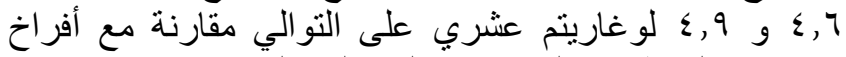

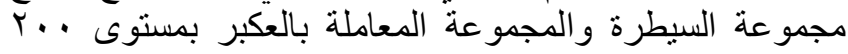
ملغم/كغ.

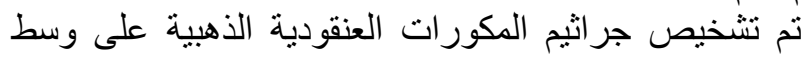
الملح وسكر المانيتول، وتم حساب عدد المكور ات التمن العنقودية الذهبية

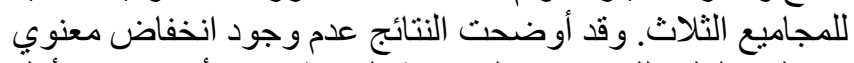

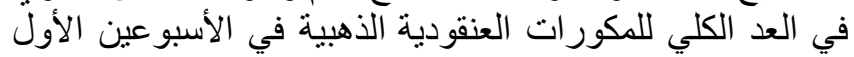

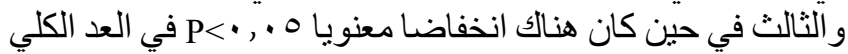

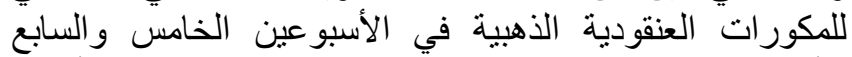

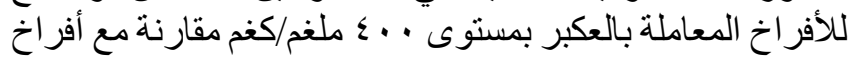

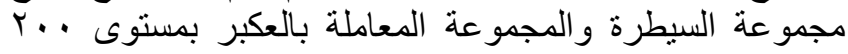

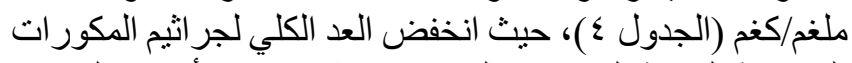

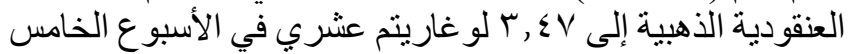

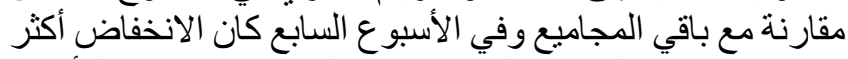

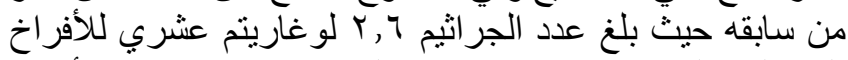

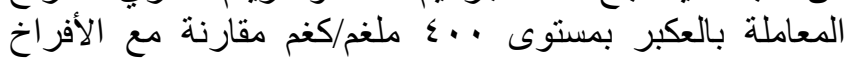

الجدول r: تأثير إضافة العكبر على العد الكلي للجراثيم في براز فروج اللحم

\begin{tabular}{|c|c|c|c|c|}
\hline الأسبوع السابع & الأسبوع الخامس & الأسبوع الثالث & الأسبو ع الأول & المجمو عة \\
\hline$a \cdot, r \cdot \pm 1 r, 7$ & $\left.a \cdot, r r_{ \pm}\right), 0$ & $a \cdot, r r \pm q, \Lambda$ & $a \cdot, 10_{ \pm} 7,0$ & السيطرة \\
\hline $\mathrm{b} \backslash \pm \mathrm{V}, 1$ & $b \cdot, 1 \pm 7,9$ & $b \cdot, 10_{ \pm}, r$ & $b \cdot, r r_{ \pm} \varepsilon, q$ & العكبر · . ب ملغم/كغم \\
\hline$c \cdot, r_{ \pm} \varepsilon, q$ & $c^{*}, Y Y_{ \pm} \varepsilon, 7$ & $c \cdot, r \cdot \pm r, q$ & $c \cdot, r_{ \pm}, r, \Lambda$ & العكبر . ـ ملغم/كغم \\
\hline
\end{tabular}

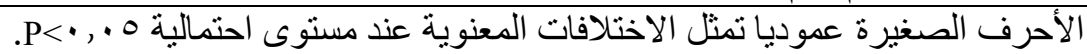

الجدول §: تأثثر إضافة العكبر على العد الكلي لجر اثيم المكورات العنقودية الذهبية في براز فروج اللحم

\begin{tabular}{|c|c|c|c|c|}
\hline الأسبوع السابع & الأسبوع الخامس & الأسبوع الثالث & الأسبوع الأول & المجمو عة \\
\hline$a \cdot, \vee \pm r, \wedge$ & $a \cdot, \cdot r \pm \xi$ & $a \cdot, \mid r \pm r, q$ & $a^{\cdot}, l \pm \varepsilon, \cdots$ & السيطرة \\
\hline$a \cdot, r \pm r, \tau$ & $a \cdot, \cdot r \pm r, \Lambda$ & $a \cdot, \wedge \pm r, \wedge \vee$ & $a \cdot, \wedge_{ \pm} \varepsilon, \cdots$ & العكبر · . r ملغم/كغم \\
\hline$b \cdot, 10 \pm r, r$ & $b \cdot, l r_{ \pm} r, \leqslant V$ & $a \cdot, \cdot r \pm r, \wedge v$ & $a \cdot, I r \pm r, q V$ & العكبر · . ع ملغم/كغم \\
\hline
\end{tabular}

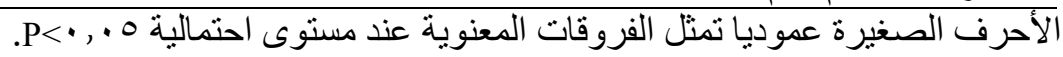

الجدول ه: تأثير إضافة العكبر على العد الكلي لجر اثيم العصيات اللبنية في براز فروج اللحم

\begin{tabular}{|c|c|c|c|c|}
\hline الأسبوع السابع & الأسبوع الخامس & الأسبوع الثالث & الأسبوع الأول & المجمو عة \\
\hline$a \cdot, Y) \pm \varepsilon, q$ & $a \cdot, l \pm \xi, \vee$ & $\mathrm{a}^{0, \wedge \pm r, q}$ & $a \cdot, r \sigma_{ \pm} r, 0$ & السيطرة \\
\hline $\mathrm{bB} \cdot, r_{ \pm} \varepsilon, l$ & $\mathrm{bB} \cdot, r_{ \pm} \varepsilon$ & $\mathrm{aA} \cdot, \mid \wedge \pm r, 0$ & $\mathrm{aA} \cdot, r) \pm r, 0$ & العكبر · . ب ملغم/كغم \\
\hline$b \cdot, r \pm r, r$ & $b \cdot, \Gamma_{ \pm} r, \Sigma V$ & $a \cdot, 1 \pm r, o v$ & $a \cdot, \mid \vee \pm r, \wedge$ & العكبر · . ـ ملغم/كغم \\
\hline
\end{tabular}

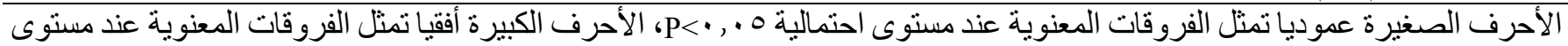

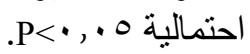


المجموعة المعاملة بالعكبر بمستوى · . مـ ملغم/كغم فكان هنالك

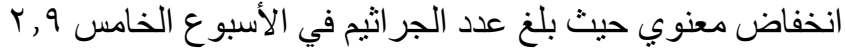

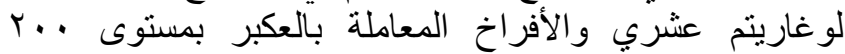

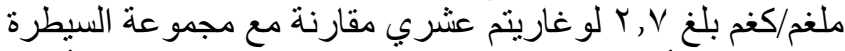

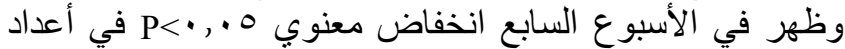

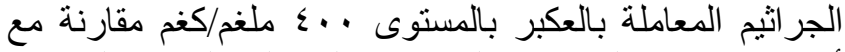

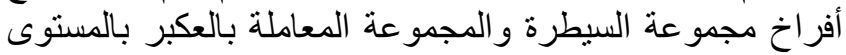

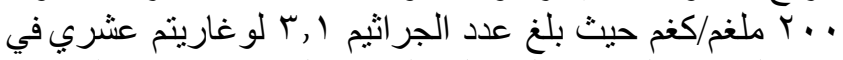

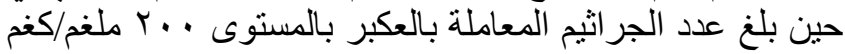

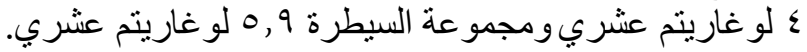

عزلت جراثيم الإيشريكيا القولونية من براز فروج اللحم الحمبر

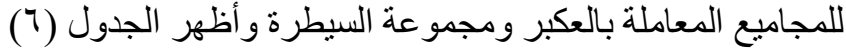

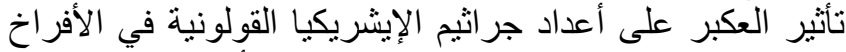

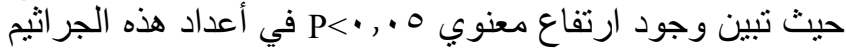

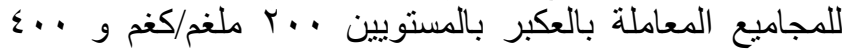

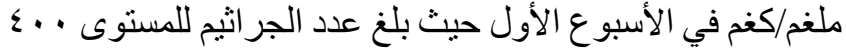

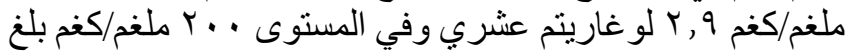

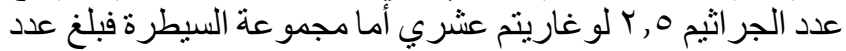

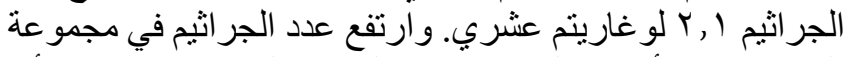

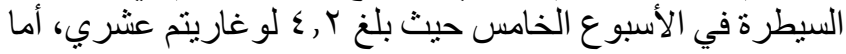
الجدول 7: تأثثير إضافة العكبر على العد الكلي لجر اثثم الإيشريكيا القولونية في براز فروج اللحم

\begin{tabular}{|c|c|c|c|c|}
\hline الأسبو ع السابع & الأسبوع الخامس & الأسبو ع الثالث & الأسبوع الأول & المجموعة \\
\hline$a \cdot, \wedge \pm 0,9$ & $a \cdot, l \pm \varepsilon, r$ & $a \cdot, \wedge \pm \Gamma, \wedge$ & $c \cdot, \Lambda_{ \pm} r, l$ & السيطرة \\
\hline$b \cdot, 1 \pm \varepsilon$ & $b \cdot, l \pm r, V$ & $b \cdot, l \pm r, q$ & $b \cdot, \wedge \pm r, o$ & العكبر · . ب ملغم/ كغم \\
\hline$c \cdot, 1 \pm r, l$ & $b \cdot, l \pm r, q$ & $\mathrm{~b} \cdot, \wedge \pm r, \wedge$ & $a \cdot, l \pm r, q$ & العكبر · ـ ملغم/ كغم \\
\hline
\end{tabular}

العكبر على تحلل الجر اثيم وتثبيط تصنيع البروتين من خلال تثنيطه

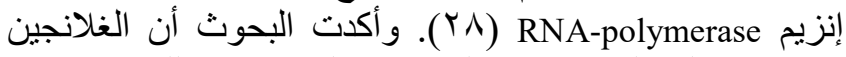

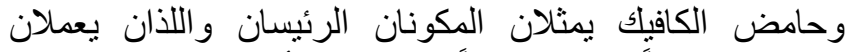

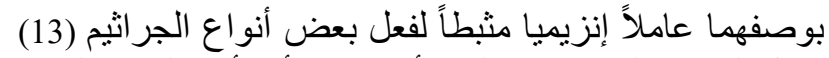

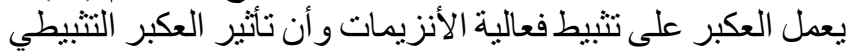

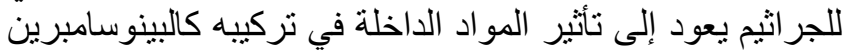

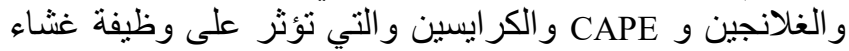

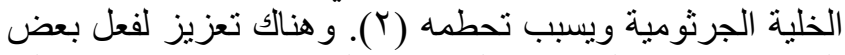

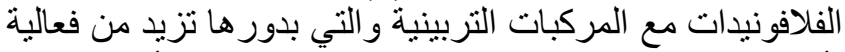

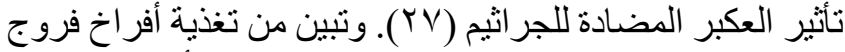

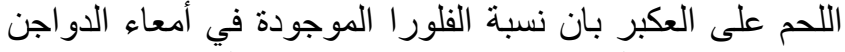

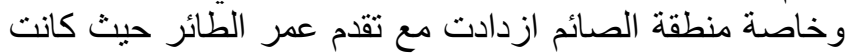

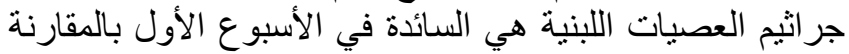

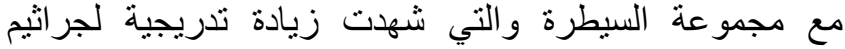

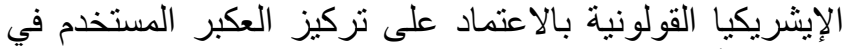

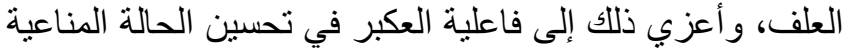

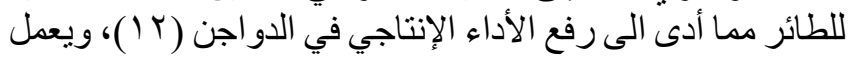

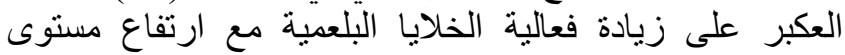

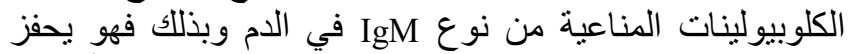

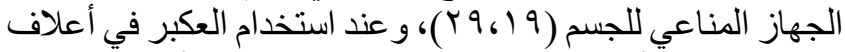

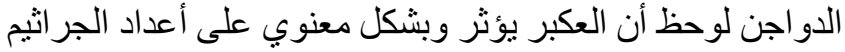

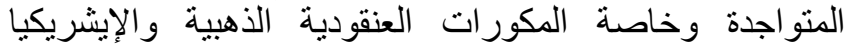

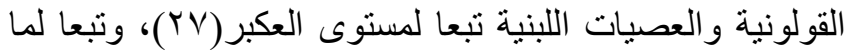
يحتويه العكبر من والتربينات البينوسامبرين و البينوبانكسين البكين وكر ايسين و الغلانجين وحامض الكافيئل وحامض الفريولين ولكين و التيني
المناقشة

تبين من نتائج التجربة وجود انخفاضا في مستوى كل من النيات

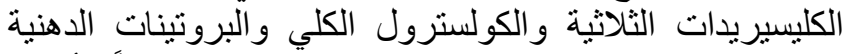

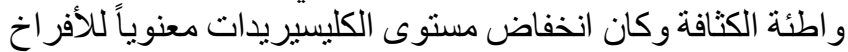

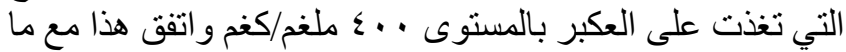

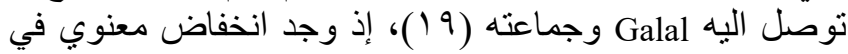

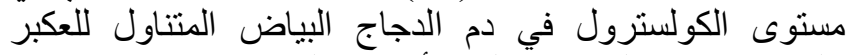

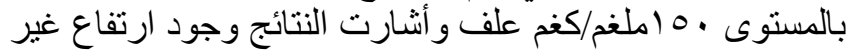

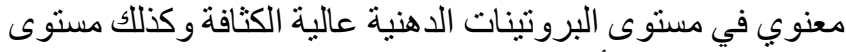

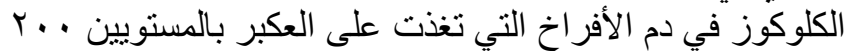

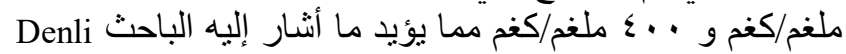

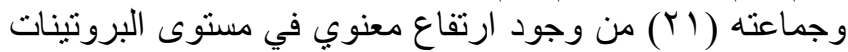

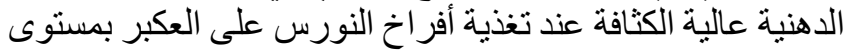

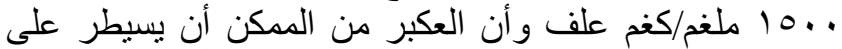

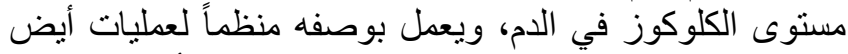

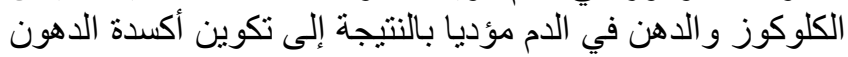

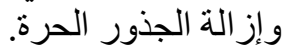

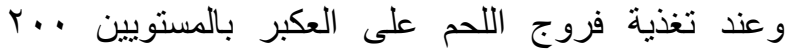

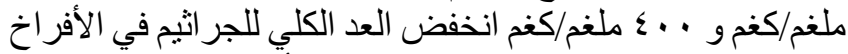

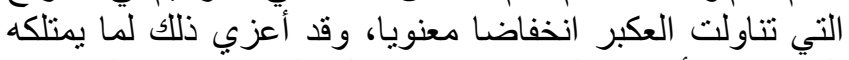

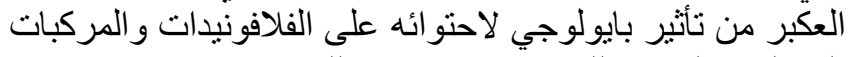

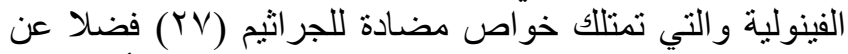

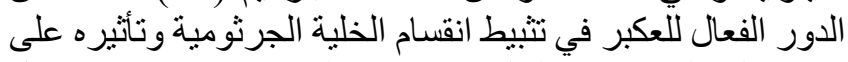

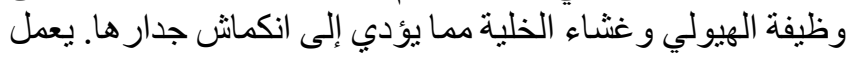




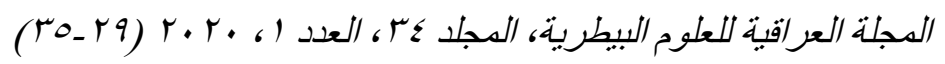

activities. J Ethnopharmacol. 2001;74(2):105-112. DOI: 10.1016/s0378-8741(00)00326-3

3. Hegazi AG, Abd El Hady FK, Abd Allah FAM. Chemical composition and antimicrobial activity of European propolis. Z Naturforsch. 2000;55:71-75. DOI: 10.1515/znc-2000-1-214

4. Marcucci MC. Propolis: Chemical composition, biological properties and therapeutic activity. Apidologie. 1995;(26):83-99. DOI: 10.1051/apido:19950202

5. Pietta PG, Gardana C, Pietta AM. Analytical methods for quality control of propolis. Fitoterapi. 2002;(1):S7-S20. DOI: 10.1016/s0367326x(02)00186-7

6. Pires G, da Silva R, Fabiana C, Luciane R. Antimicrobial activity of two Brazilian commercial propolis extracts. Brazilian J Oral Sci. 2006;5(16):967-970. https://doi.org/10.20396/bjos.v5i16.8641876

7. Fernandes A, Balestrin EC, Betoni JE, Orsi Rde O, da Cunha Mde L, Montelli AC. Propolis: Anti- Staphylococcus aureus activity and synergism with antimicrobial drugs. Mem Inst Oswaldo Cruz. 2005;100(5):563-566. DOI: 10.1590/s0074-02762005000500018

8. Stepanovic S, Antic N, Dakic I, Svabic M. In vitro antimicrobial activity of propolis and synergism between propolis and antimicrobial drugs. Microbiol Res. 2003;158:353-357. DOI: 10.1078/0944-501300215

9. Yaghobi SMJ, Ghorbani GR, Soleimanian S, Satari R. Antimicrobial activity of Iranian propolis and its chemical composition. DARU. 2007;15(1):45-48.

10. Gonsales GZ, Orsi RO, Fernandes JA, Rodrigues P, Funari SRC. Antibacterial activity of propolis collected in different regions of Brazil. J Venom Anim Toxins Incl Trop Dis. 2006;12(2):276-284. doi.org/10.1590/S1678-91992006000200009

11. Haddadin MSY, Nazer I, Sara JR, Robinson RK. Effect of propolis on two bacterial species with probiotic potential. Pakistan J Nutr. 2008;7(2):391-394. DOI: 10.3923/pjn.2008.391.394

12. Krocko, M., M. Canigova, J. Bezekova, M. Lavova, P. Hascik and V. Duckova,. Effect of nutrition with propolis and bee pollen supplements on bacteria colonization pattern in gastrointestinal tract of broiler chickens. Anim. Sci. Biotechnol., 2012;45(1):63-67.

13. Farre R, Frasquet I, Sanchez A. Propolis and human health. Ars Pharmaceutica. 2004;45(1):21-43.

14. Usami E, Kusano G, Takayose T, Wachi H, Seyama Y. Assessment of antioxidant activity of natural compounds by water and lipid -soluble antioxidant factor. Yakugaku Zasshi. 2004;124:847-850. DOI: 10.1248/yakushi. 124.847

15. Sun F, Hayami S, Haruna S, Ogiri Y, Tanaka K, Yamada Y. In vivo antioxidative activity of propolis evaluated by the interaction with vitamins $\mathrm{C}$ and $\mathrm{E}$ and the level of lipid hydroperoxides in rats. J Agric Food Chem. 2000;48(5):1462-1465. DOI: 10.1021/jf990594t

16. Shalmany KS, Shivazad M. The effect of diet propolis supplementation on Ross broiler chicks performance. Inter J Poul Sci. 2006;5(1):84-88. DOI: 10.3923/ijps.2006.84.88

17. Biavatti MW, Bellaver MH, Volpato L, Bellaver C. Preliminary studies of alternative feed additives for broilers: Alternanthera Brazilian extract, propolis extract and linseed oil. Rev Bras Cienc Avic. 2003;(5):147-151. doi.org/10.1590/S1516-635X2003000200009

18. Abdullah TA, Hassan MG. The effect of propolis feed supplementation on hygiene and performance of broiler chickens. Iraqi J Vet Sci. 2011;25(2):77-82..doi.org/10.33899/ijvs.2011.5647

19. Galal A, Abd El-Motaal AM, Ahmed AMH, Zaki TG. Productive performance and immune response of laying hens as affected by dietary propolis supplementation. Inter J Poul Sci. 2008;7(3):272-278. DOI: 10.3923/ijps.2008.272.278

20. Tatli P, Seven I, Yilmaz M, Simsek UG. The effects of Turkish propolis on growth and carcass characteristics in broilers under heat stress. Anim FeedSciTechnol.2007;146(1):137-148.DOI:10.1016/j.anifeedsci.2007. 11.003

21. Denli M, Cankaya S, Silici S, Okan F, Uluocak AN. Effect of dietary addition of Turkish propolis on the growth performance, carcass characteristics and serum variables of quail (Coturnix coturnix
من شأنها أن تؤثر على الفلورا المعوية لفروج اللحم (·r).

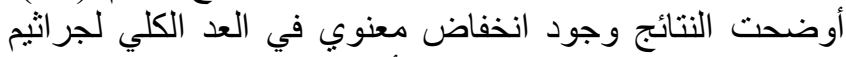

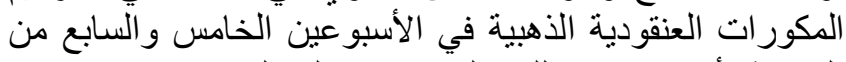

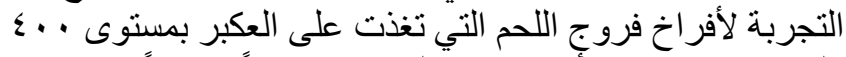

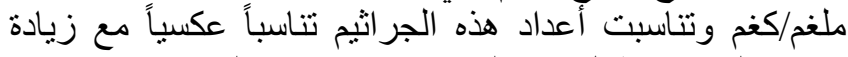

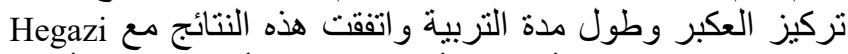

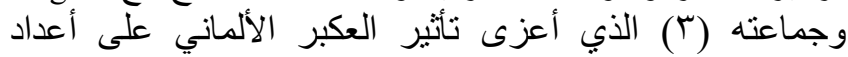

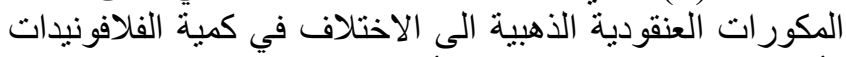

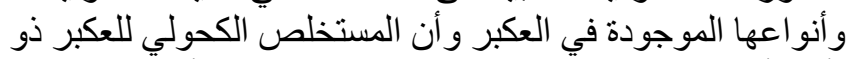

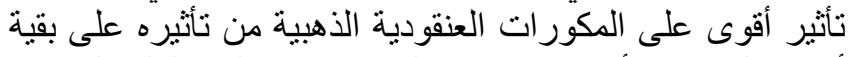

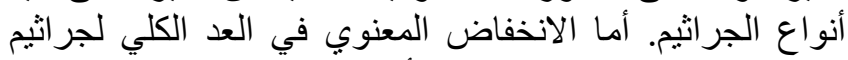

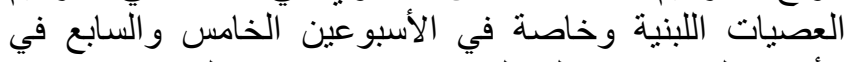

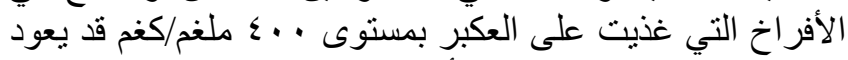

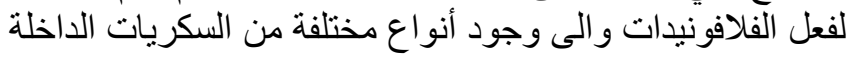

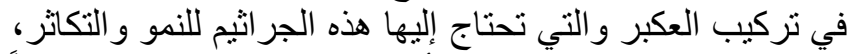

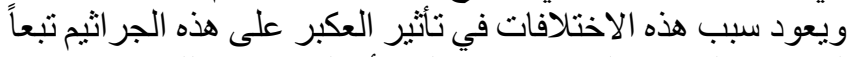

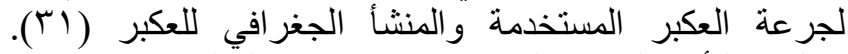

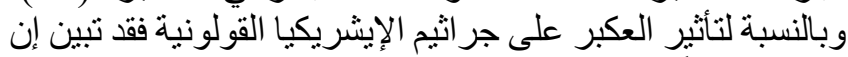

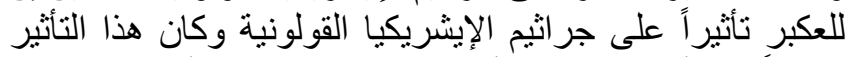

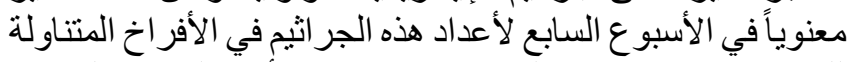

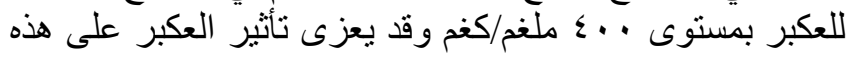

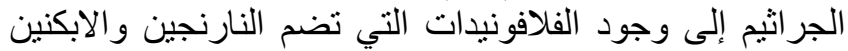

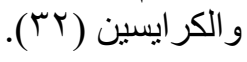

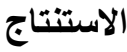

نستتتج من البحث أهمية استخدام العكبر كمعزز غذائي في ألهاي

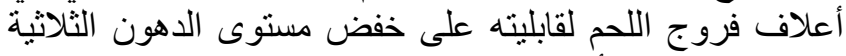

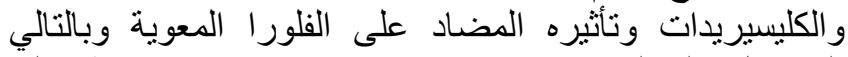

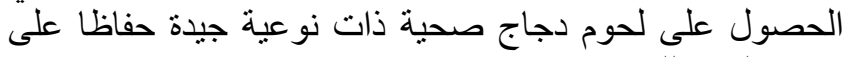
صحة المستهلك.

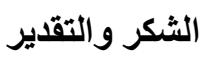

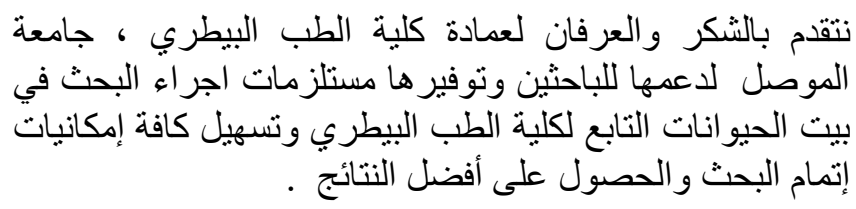
المصادر

1. Castaldo S, Capasso F. Propolis, an old remedy used in modern medicine. Fitoterapia. 2002;(1):51-56. DOI: 10.1016/s0367$326 x(02) 00185-5$

2. Marcucci MC, Ferreres F, Garcia C, Bankova VS, DeCastro SL, Dantas AP. Phenolic compounds from Brazilian propolis with pharmacological 


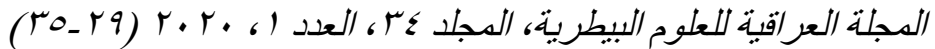

28. Kartal M, Yildiz S, Kaya S, Kurucu S, Topcu G. Antimicrobial activity of propolis samples from two different regions of Anatolia. J Ethnopharmacol. 2003;86(1):69-73. DOI: $\quad 10.1016 / \mathrm{s} 0378$ 8741(03)00042-4

29. Orsi RO, Funari SRC, Soares AVC, Calvi SA, Oliveira SL, Sforcin JM, Bankova V. Immune modulatory action of propolis on macrophage activation. J Venom Anim Toxins. 2000;6(2):205-219. doi.org/10.1590/S0104-79302000000200006.

30. Bankova V, Popova M, Bogdanov S, Sabatini A. Chemical composition of European propolis: Expected and unexpected results. Z Naturforsch C. 2002;57(5-6):530-533. DOI: 10.1515/znc-2002-5-622

31. Abd El Hady FK, Hegazi AG. Egyptian propolis: 2. Chemical composition, antiviral and antimicrobial activities of east Nile delta propolis. Z Naturforsch C. 2002;56(3-4):386-394. DOI: 10.1515/znc2002-3-431

32. Hikmet K, Nazime M. Antimicrobial activity and chemical compositions of Turkish propolis from different region. Afr J Biotech. 2006;5(11):1151-1153. japonica). Asian-Australasian J Anim Sci. 2005;18(6):848-854. DOI: https://doi.org/10.5713/ajas.2005.848

22. Kaneko JJ, Harvey JW, Bruss ML: Clinical biochemistry of domestic animals $6^{\text {th }}$ ed. Amsterdam,Netherlands: Elsevier/Academic Press; 2008;90-95. ISBN: 9780080568829.

23. Baron EJ, Finegold SM. Diagnostic microbiology. $8^{\text {th }}$ ed. USA: Mosby Company; 1994. 438-443 p. ISBN 10: 0801603447

24. Quinn PJ, Carter ME, Markery B, Carter GR. Clinical veterinary microbiology. $1^{\text {st }}$ ed. London: Elsevier Limited; 2004. 118-126 p. ISBN10 0723417113

25. MacFaddin P. Biochemical tests for identification of medical bacteria. $3^{\text {rd }}$ ed. Philadelphia: Lippincott Williams and Wilkins; 2000. 825-826 p.

26. SAS. SAS user guide statistics version 6.12. USA: Cary Inc; 2001. ISBN 1-59047-243-8

27. Kosalec I, Pepelnijak S, Bakmaz M, Wladimir V. Flavonoid analysis and antimicrobial activity of commercially available propolis products. Acta Pharm. 2005;55(4):423-430.

- $\quad$ https://orcid.org/ 0000-0001-7250-0117 للباحثة د.منتهـ 